PROCEEDINGS OF THE

AMERICAN MATHEMATICAL SOCIETY

Volume 128, Number 12, Pages 3715-3721

S 0002-9939(00)05488-5

Article electronically published on June 7,2000

\title{
PERTURBED DOLBEAULT OPERATORS AND THE HOMOLOGY TODD CLASS
}

\author{
JEFFREY FOX AND PETER HASKELL
}

(Communicated by Jozef Dodziuk)

\begin{abstract}
This paper discusses the role played by perturbed Dolbeault operators in relating the coherent sheaf and elliptic operator perspectives on the $K$ homology of projective varieties. Among the consequences are index formulas for perturbed Dolbeault operators.
\end{abstract}

In this paper we make some observations about the index theory of perturbed Dolbeault operators, with particular attention to the role of the homology Todd class [2], 3] in this index theory. A perturbed Dolbeault operator is the sum of a Dolbeault operator and a vector-bundle map (the perturbation) that is invertible off some compact subset of the underlying manifold. We focus on the following setting. Let $s$ be a meromorphic section of a holomorphic vector bundle over a smooth projective algebraic variety $V$. Assume that the closure of the section's zero locus has empty intersection with the closure of the section's polar locus. Let $M$ be the complement in $V$ of the section's polar locus. Over $M$ use the section and all exterior powers of the holomorphic vector bundle's dual to form a Koszul complex. Assemble this complex into a two-term complex consisting of a vectorbundle map from the direct sum of exterior powers of even degree to the direct sum of exterior powers of odd degree. This vector-bundle map is invertible away from the original section's zero locus. We use it to provide a perturbation for the Dolbeault operator on $M$.

In the first section we present conditions, for the most part involving the interaction of the perturbation with the metric chosen for $M$, that ensure that a perturbed Dolbeault operator represents a $K$ homology class for $V$ and that this $K$ homology class is the Kasparov product of a $K$ theory class represented by the perturbation and a $K$ homology class represented by the Dolbeault operator. This product result permits the use of standard characteristic class techniques in calculating the homology Chern character and hence the index of the perturbed Dolbeault operator. If the section $s$ is regular [7], the perturbed Dolbeault operator's $K$ homology class equals that determined by the structure sheaf of $s$ 's zero scheme. It follows that the perturbed Dolbeault operator's homology Chern character is the homology Todd

Received by the editors February 4, 1999.

2000 Mathematics Subject Classification. Primary 58J20, 19L10, 19K35.

Key words and phrases. Perturbed Dolbeault operator, homology Todd class, homology Chern character.

The first author's work was supported by the National Science Foundation.

The second author's work was supported by the National Science Foundation under Grant No. DMS-9800782. 
class of this sheaf. In the second section we reverse perspective to show that for a given section $s$, it is possible to choose a metric on $M$ so that the Dolbeault operator perturbed by $s$ 's assembled Koszul complex satisfies the conditions presented in the first section. These results establish perturbed Dolbeault operators as a natural link between the view of $K$ homology based on coherent algebraic sheaves and the view based on elliptic operators.

\section{The Product: Conditions And implications}

In this section we present conditions that allow us to analyze a perturbed Dolbeault operator as a Kasparov product. We also discuss the implications of this "factorization" of the perturbed Dolbeault operator. We begin the section by setting notation and describing our objects of study.

Let $V$ be a projective algebraic variety, and let $Y$ be a codimension-one subvariety. Denote $V \backslash Y$ by $M . M$ retains the complex structure it inherits from $V$, but we assume $M$ has been given a complete Hermitian metric with associated canonical connection. Let $E$ be a Hermitian holomorphic vector bundle, with associated canonical connection, over $M$, and let $s$ be a holomorphic section of $E$. In this section we do not assume that $E$ is defined over all of $V$.

The Dolbeault complex on $M$ is formed using the differential forms of type $(0, q)$ on $M$ and the operator $\bar{\partial}$. This can be assembled to yield an operator $\bar{\partial}+\bar{\partial}^{*}$ from sections of $S^{ \pm}$to sections of $S^{\mp}$. Here

$$
S^{+}=\bigoplus_{k} \bigwedge^{0,2 k} T_{\mathbb{C}}^{*}(M)
$$

and

$$
S^{-}=\bigoplus_{k} \bigwedge^{0,2 k+1} T_{\mathbb{C}}^{*}(M)
$$

One can think of $\bar{\partial}$ as defined on smooth compactly supported forms and of $\bar{\partial}^{*}$ as the formal adjoint of $\bar{\partial}$, but because $M$ is complete, $\bar{\partial}+\bar{\partial}^{*}$ has on $L^{2}$ forms a unique closed extension, which we also denote $\bar{\partial}+\bar{\partial}^{*}$. When $S^{ \pm}$is tensored with an auxiliary vector bundle with connection, analogous reasoning leads to operators that we also call $\bar{\partial}+\bar{\partial}^{*}$.

We use $\wedge^{k} E^{*}$ to denote the fiberwise exterior powers of the vector bundle dual to $E$. For each $k$ there is a map

$$
S: \bigwedge^{k} E^{*} \rightarrow \bigwedge^{k-1} E^{*}
$$

defined by

$$
S\left(\epsilon_{1} \wedge \ldots \wedge \epsilon_{k}\right)=\sum_{j=1}^{k}(-1)^{j+1} \epsilon_{j}(s) \epsilon_{1} \wedge \ldots \wedge \widehat{\epsilon}_{j} \wedge \ldots \wedge \epsilon_{k} .
$$

Let $n$ denote the dimension over $\mathbb{C}$ of $E$ 's fibers. The maps $S$ fit together to define a complex

$$
0 \rightarrow \bigwedge^{n} E^{*} \rightarrow \bigwedge^{n-1} E^{*} \rightarrow \ldots \rightarrow \bigwedge^{0} E^{*} \rightarrow 0
$$


that is exact at every point where $s$ takes a nonzero value. This complex assembles to define a vector-bundle map

$$
S+S^{*}: \bigoplus_{k \text { even }} \bigwedge^{k} E^{*} \rightarrow \bigoplus_{j \text { odd }} \bigwedge^{j} E^{*}
$$

which we denote

$$
A: E_{0} \rightarrow E_{1} \text {. }
$$

The Hermitian holomorphic structure on $E$ defines Hermitian holomorphic structures and thus associated connections on $E_{0}$ and $E_{1}$. When the vector bundles $E_{i}$ are tensored with the bundles $S^{ \pm}$, we extend the vector-bundle map $A$ to the tensor products by using the identity in the additional factor. We continue to use the notation $A$ for this extended operator.

The perturbed Dolbeault operator we study is

$$
D_{A}=\left(\begin{array}{cc}
A & -\left(\bar{\partial}+\bar{\partial}^{*}\right) \\
\bar{\partial}+\bar{\partial}^{*} & A^{*}
\end{array}\right)
$$

mapping $L^{2}$ sections of $E_{0} \otimes S^{+} \oplus E_{1} \otimes S^{-}$to $L^{2}$ sections of $E_{1} \otimes S^{+} \oplus E_{0} \otimes S^{-}$. Here again the operator can be defined first on smooth compactly supported sections, but it has a unique closed extension, which is what $D_{A}$ denotes.

Notation. For an operator $\gamma$, let $q(\gamma)$ denote the operator

$$
\left(\begin{array}{cc}
0 & \gamma^{*} \\
\gamma & 0
\end{array}\right)
$$

For $C^{*}$ algebras $A$ and $B$ our approach to $K K(A, B)$ is based on unbounded Kasparov $A-B$ bimodules $(\mathcal{E}, T)$, the set of which is denoted $\Psi(A, B)$. Here $T$ is an unbounded operator, satisfying certain conditions, on the Hilbert $B$-module $\mathcal{E}$. Among our conventions are: functions act on sections of vector bundles by pointwise multiplication; and a function-valued inner product on sections of a vector bundle arises from pointwise application of a Hermitian inner product. The class in $K K(A, B)$ represented by $(\mathcal{E}, T)$ is denoted by $[(\mathcal{E}, T)]$ or $[T]$. The use of unbounded Kasparov bimodules to represent $K K$ classes is due to [1]. The general expression of the Kasparov product in terms of unbounded bimodules is due to [13. The following theorem is a special case of a theorem proven in $[8$.

Theorem 1.1. Suppose that $D_{A}$ is a perturbed Dolbeault operator of the type described above. Suppose also that the following conditions are satisfied.

(1) For every $f \in C^{\infty}(V)$ and every vector field $X$ on $M$ having pointwise norm everywhere no greater than one, $\nabla_{X} f$ is bounded on $M$.

(2) The infima of the pointwise spectra of $A^{*} A(x)$ and $A A^{*}(x)$ go to infinity as $x$ goes to infinity on $M$.

(3) There is a positive constant $k$ such that off some compact subset of $M$,

$\left(\begin{array}{cc}A^{*} A & \left(\bar{\partial}+\bar{\partial}^{*}\right) \circ A^{*}-A^{*} \circ\left(\bar{\partial}+\bar{\partial}^{*}\right) \\ A \circ\left(\bar{\partial}+\bar{\partial}^{*}\right)-\left(\bar{\partial}+\bar{\partial}^{*}\right) \circ A & A A^{*}\end{array}\right) \geq k \cdot\left(\begin{array}{cc}A^{*} A & 0 \\ 0 & A A^{*}\end{array}\right)$

and

$\left(\begin{array}{cc}A A^{*} & A \circ\left(\bar{\partial}+\bar{\partial}^{*}\right)-\left(\bar{\partial}+\bar{\partial}^{*}\right) \circ A \\ \left(\bar{\partial}+\bar{\partial}^{*}\right) \circ A^{*}-A^{*} \circ\left(\bar{\partial}+\bar{\partial}^{*}\right) & A^{*} A\end{array}\right) \geq k \cdot\left(\begin{array}{cc}A A^{*} & 0 \\ 0 & A^{*} A\end{array}\right)$. 
Then

$$
\left(L^{2}\left(E_{0} \otimes S^{+} \oplus E_{1} \otimes S^{-}\right) \oplus L^{2}\left(E_{1} \otimes S^{+} \oplus E_{0} \otimes S^{-}\right), q\left(D_{A}\right)\right) \in \Psi(C(V), \mathbb{C}),
$$

and $\left[q\left(D_{A}\right)\right]$ equals the Kasparov product $[q(A)] \otimes_{C_{0}(M)}\left[\bar{\partial}+\bar{\partial}^{*}\right]$ of $[q(A)] \in$ $K K\left(C(V), C_{0}(M)\right)$ and $\left[\bar{\partial}+\bar{\partial}^{*}\right] \in K K\left(C_{0}(M), \mathbb{C}\right)$.

Proof. Because a more general theorem is proven in [8] and a special case involving Dolbeault operators is proven in [9], we give only an outline of the proof. Conditions (2) and (3) imply that $q\left(D_{A}\right)^{2}$ is the sum of a second-order elliptic differential operator and a vector-bundle map the infimum of whose pointwise spectrum goes to infinity as one goes to infinity on $M$. From this it follows that $\left(1+q\left(D_{A}\right)^{2}\right)^{-1}$ is compact. Condition (1) implies that the commutator of $q\left(D_{A}\right)$ with each $f \in C^{\infty}(V)$ is a bounded operator. By $[1]$ these and more straightforward observations imply that $\left(L^{2}\left(E_{0} \otimes S^{+} \oplus E_{1} \otimes S^{-}\right) \oplus L^{2}\left(E_{1} \otimes S^{+} \oplus E_{0} \otimes S^{-}\right), q\left(D_{A}\right)\right) \in \Psi(C(V), \mathbb{C})$. In order to use [13] to show that this Kasparov bimodule represents that asserted product, note that: conditions (2) and (3) imply that the domain of $q\left(D_{A}\right)$ is contained in the domain of $q(A) \otimes I$; the same conditions establish the semiboundedness required by [13]; and smooth compactly supported sections lead to the bounded commutators required by the connection condition.

The representation of the perturbed Dolbeault operator as this Kasparov product has various implications. Viewing the Dolbeault operator's class in terms of the $K$ theory class on $T^{*} M$ represented by the Dolbeault operator's symbol, we can use excision in $K$ theory to conclude that the perturbed Dolbeault operator's class is determined by data supported in an arbitrarily small neighborhood of the section $s$ 's zero locus. Also the homology Chern character of the perturbed Dolbeault operator is the Poincaré dual of the cup product of the perturbation's Chern character and the manifold M's Todd class. These classes can be calculated using Chern-Weil theory or other standard techniques. Finally the perturbed Dolbeault operator's class is the $K$-theoretic Poincare dual of the perturbation's $K$ theory class.

If the section $s$ is regular (as defined below), then the Koszul complex is a resolution of the structure sheaf of $s$ 's zero scheme [7]. In this situation this sheaf is an algebraic realization of the $K$ homology class $K$-theoretically Poincaré dual to the the Koszul complex. It follows that the perturbed Dolbeault operator represents the $K$ homology class determined by this sheaf. Also the homology Chern character of the perturbed Dolbeault operator is the homology Todd class [2], [3] of this sheaf. Algebro-geometric properties of the homology Todd class then have implications for the index theory of the perturbed Dolbeault operator: the index is invariant in flat families; the homology Todd class of a local complete intersection can be calculated from the Todd class of the virtual tangent bundle; and the Porteous formula [14] can be used to relate the homology Todd class of a singular variety to that of its desingularization. In this context we note that in giving a formula for the difference of domain and target $L$-genera for a stratified map, S. Cappell and J. Shaneson [5] mention that they will discuss the Todd class in later work.

Definition (from [7]). In a neighborhood of each point $x$ of the section $s$ 's zero set, choose a local trivialization of $E$. Denote the associated component functions of $s$ by $f_{x, 1}, \ldots f_{x, n}$, and view these as elements of $\mathcal{O}_{x}$. The section $s$ is called regular if at each $x$ in its zero set: the ideal generated by $\left\{f_{x, 1}, \ldots f_{x, n}\right\}$ is not $\mathcal{O}_{x} ; f_{x, 1}$ is 
not a zero-divisor in $\mathcal{O}_{x}$; and for each $m$, the image of $f_{x, m}$ is not a zero-divisor in $\mathcal{O}_{x} /\left(f_{x, 1}, \ldots, f_{x, m-1}\right)$.

\section{A CHOICE OF METRIC}

In this section we show how to proceed from a meromorphic section to a perturbed Dolbeault operator that lives on the complement of the section's polar divisor, that has the section's Koszul complex as its perturbation, and that satisfies the conditions of Theorem 1.1. By the discussion in the previous section, if the section $s$ is regular, this perturbed Dolbeault operator then represents the $K$ homology class determined by the structure sheaf of $s$ 's zero scheme. The key to establishing the properties we need is choosing an appropriate complete Kähler metric on the complement of the polar divisor. Our general construction uses a finite-volume metric. Motivated by [4], 12], we previously [9] used an infinite-volume metric in special cases.

Let $V$ be a projective algebraic variety and let $Y$ be a divisor on $V$. $Y$ need not be smooth, but we assume that $Y$ has at worst normal crossings and that each irreducible component of $Y$ has multiplicity one. Let $M=V \backslash Y$. We follow [15], which follows [6], in observing that $M$ can be given the complete Kähler metric associated with the following Kähler form:

$$
c \omega-\sum_{j} \partial \bar{\partial} \log \left(\log ^{2}\left(\left|\sigma_{j}\right|^{2}\right)\right) .
$$

Here $\omega$ is the Kähler form inherited by $V$ from the ambient projective space, and $c$ is a sufficiently large positive constant. Also $j$ indexes the irreducible components of $Y, Y=\bigcup_{j \in J} Y_{j}$. For each $j, \sigma_{j}$ is the section defining $Y_{j}$ of the line bundle associated with $Y_{j}$, and $\left|\sigma_{j}\right|$ refers to a pointwise norm coming from a Hermitian structure on the line bundle.

Theorem 2.2. Let $E$ be a Hermitian holomorphic vector bundle, with associated canonical connection, on the projective algebraic variety $V$. Let $s$ be a meromorphic section of $E$. Assume that the closures of the zero and polar loci of $s$ do not intersect and that the polar divisor has at worst normal crossings. Denote the polar divisor by $\sum_{j \in J} n_{j} Y_{j}$, where the $Y_{j}$ are distinct irreducible divisors and each $n_{j}>0$. Give $M=V \backslash Y$ the metric determined by $Y=\bigcup_{j \in J} Y_{j}$ and the Kähler form (2.1). Give $\left.E\right|_{M}$ the restrictions of its structures on $V$. Let $D_{A}$ be the perturbed Dolbeault operator constructed on $M$ by using the metric just assigned and by using the assembled Koszul complex of $\left.s\right|_{M}$ as the perturbation. Then this metric on $M$ and this perturbed Dolbeault operator $D_{A}$ satisfy the conditions of Theorem 1.1.

Proof. To prove that the conditions of Theorem 1.1 are satisfied in this setting, we focus on analysis "at infinity" on $M$ in the metric introduced in this section. Our analysis "at infinity" on $M$ is often expressed in terms of coordinates on $V$ around a point $p \in Y$. For $p \in Y$, let $J_{p}=\left\{j \in J: p \in Y_{j}\right\}$. Choose a trivialization of $E$ around $p$ over a coordinate chart whose intersection with $Y$ has closure contained in $\bigcup_{j \in J_{p}} Y_{j}$ and which does not intersect the zero set of $s$. Choose coordinates in which $p$ corresponds to the origin, in which the coordinates $z_{j}, j \in J_{p}$, are such that $Y_{j}$ corresponds to $z_{j}=0$, and in which $z_{k}, k \in K_{p}$, denote the remaining coordinates. We sometimes use real $x, y$-coordinates or polar $r, \theta$-coordinates in 
place of complex $z$-coordinates. In our neighborhood of $p$, the metric behaves like

$$
\sum_{j \in J_{p}} \frac{1}{r_{j}^{2} \cdot \log ^{2}\left(r_{j}^{2}\right)}\left(d r_{j} \otimes d r_{j}+r_{j}^{2} d \theta_{j} \otimes d \theta_{j}\right)+\sum_{k \in K_{p}}\left(d x_{k} \otimes d x_{k}+d y_{k} \otimes d y_{k}\right) .
$$

Note that if $f$ is a function that is smooth on $V$, then for each $j,\left(1 / r_{j}\right) \nabla_{\partial / \partial \theta_{j}} f$ is bounded on $M$. Condition (1) of Theorem 1.1 follows directly from this observation and the above description of the metric.

In a neighborhood of $p$, the section $s$ is the product of

$$
\prod_{j \in J_{p}} z_{j}^{-n_{j}}
$$

and a holomorphic section that does not vanish at $p$. Thus in this neighborhood $A^{*} A$ and $A A^{*}$ are positive multiples of $\prod_{j \in J_{p}}\left|z_{j}\right|^{-2 n_{j}}$. This implies condition (2) of Theorem 1.1. We can combine the observation that if $h$ is a section that is smooth on $V$, then for each $j,\left(1 / r_{j}\right) \nabla_{\partial / \partial \theta_{j}} h$ is bounded on $M$ with the description of the metric we are using to see that if $X$ is a unit vector field on $M$, then the norm of $\nabla_{X} s$ is bounded above, in a neighborhood of $p$, by a constant multiple of

$$
\left(\prod_{j \in J_{p}} r_{j}^{-n_{j}}\right) \cdot\left(\sum_{j \in J_{p}}\left|\log \left(r_{j}^{2}\right)\right|\right) .
$$

This and the description of $A^{*} A$ and $A A^{*}$ from the beginning of this paragraph imply condition (3) of Theorem 1.1.

Corollary 2.3. Make the same assumptions as in Theorem 2.2 except drop the assumption that the polar divisor of $s$ has at worst normal crossings. Then it is possible to give the complement of s's polar divisor a complete Kähler metric so that this metric and the perturbed Dolbeault operator on this complement constructed from $E$ with restricted structures and from the assembled Koszul complex of s's restriction satisfy the conditions of Theorem 1.1.

Proof. Use embedded resolution [11, see, e.g., 10] of the singularities of $s$ 's polar divisor to create $\pi: \widetilde{V} \rightarrow V$ in which $\pi^{-1}(Y)$ has at worst normal crossings. The metric constructed in this section for $\widetilde{V} \backslash \pi^{-1}(Y)$ descends to define a metric on $V \backslash Y$. The calculations needed to establish the conditions of Theorem 1.1 reduce to calculations on $\widetilde{V} \backslash \pi^{-1}(Y)$, where Theorem 2.2 shows that they give the desired results. In essence we create a $K$ homology class on $V$ that is the image under $\pi_{*}$ of a $K$ homology class on $\widetilde{V}$ that can be analyzed by Theorem 2.2 .

Suppose one wishes to start with a given structure sheaf and proceed to construct an associated perturbed Dolbeault operator. Because our construction is based on a meromorphic section, it supports the use of the simplest possible vector bundles in constructing from the structure sheaf the perturbed Dolbeault operator. It can even be possible to make use of a section whose zero and polar loci have intersecting closures. For instance the cuspidal cubic curve in $\mathbb{C P}^{2}$ is the zero set of the function (or section of the trivial line bundle) $x^{2}-y^{3}$ defined on the set where $z \neq 0$. One can blow up the intersection of the closures of the zero and polar loci sufficiently many times to separate them. Because the closure of the zero locus is smooth at the original intersection point, the resulting variety and function become the foundation for using the above constructions to represent the $K$ homology class of 
the cuspidal cubic's structure sheaf. We note in passing that after passing to the assembled Koszul complex, one can choose to do the perturbed Dolbeault operator construction over $V$ rather than $M$. Use the Dolbeault operator on $V$ to replace the Dolbeault operator on $M$. For the auxiliary vector bundles, note that $E$ and the constructions done with it extend over $V$. Then form $\widehat{E_{0}}$ by extending $E_{0}$ from $V$ to $M$. Form $\widehat{E_{1}}$ by using the restriction of $A^{-1}$ to a deleted neighborhood of $Y$ to clutch the vector bundles $E_{1}$ over $V$ and $E_{0}$ over a neighborhood of $Y$. The map $A$ extends to $V$ by the identity map from $E_{0}$ to $E_{0}$ over the neighborhood where both $\widehat{E_{0}}$ and $\widehat{E_{1}}$ can be identified with $E_{0}$.

\section{REFERENCES}

1. S. Baaj and P. Julg, Théorie bivariante de Kasparov et opérateurs non bornés dans les $C^{*}-$ modules hilbertiens, C. R. Acad. Sc. Paris, Série I t. 296 (1983), 875-878. MR 84m:46091

2. P. Baum, Riemann-Roch theorem for singular varieties, Proc. Sympos. Pure Math., vol. 27, part 2, Amer. Math. Soc., Providence, R.I., 1975, pp. 3-16. MR 52:10736

3. P. Baum, W. Fulton, and R. MacPherson, Riemann-Roch for singular varieties, Publ. Math. I.H.E.S. 45 (1975), 101-145. MR 54:317

4. N. V. Borisov and K. N. Ilinski, $N=2$ supersymmetric quantum mechanics on Riemann surfaces with meromorphic superpotentials, Commun. Math. Phys. 161 (1994), 177-194. MR 95g:58224

5. S. Cappell and J. Shaneson, Stratifiable maps and topological invariants, J. Amer. Math. Soc. 4 (1991), 521-551. MR 92d:57024

6. M. Cornalba and P. Griffiths, Analytic cycles and vector bundles on non-compact algebraic varieties, Invent. Math. 28 (1975), 1-106. MR 51:3505

7. W. Fulton and S. Lang, Riemann-Roch Algebra, Springer-Verlag, New York, 1985. MR 88h:14011

8. J. Fox, C. Gajdzinski, and P. Haskell, Homology Chern characters of perturbed Dirac operators, Houston J. Math., to appear.

9. J. Fox and P. Haskell, Index theory of perturbed Dolbeault operators: smooth polar divisors, Internat. J. Math., to appear.

10. R. Hartshorne, Algebraic Geometry, Springer-Verlag, New York, 1977.

11. H. Hironaka, Resolution of singularities of an algebraic variety over a field of characteristic zero. I, II, Annals of Math. 79 (1964), 109-203, 205-326. MR 33:7333

12. S. Klimek and A. Lesniewski, Local rings of singularities and $N=2$ supersymmetric quantum mechanics, Commun. Math. Phys. 136 (1991), 327-344. MR 92i:32037

13. D. Kucerovsky, The KK-product of unbounded modules, K-Theory 11 (1997), 17-34. MR 98k:19007

14. I. R. Porteous, Blowing up Chern classes, Proc. Cambridge Phil. Soc. 56 (1960), 118-124. MR 22:12543

15. M. Stern, Index theory for certain complete Kähler manifolds, J. Differential Geom. 37 (1993), 467-503. MR 94g:58212

Department of Mathematics, University of Colorado, Boulder, Colorado 80309

E-mail address: jfox@euclid.colorado.edu

Department of Mathematics, Virginia Polytechnic Institute and State University, BLACKSBURG, ViRginia 24061

E-mail address: haskell@math.vt.edu 